\title{
Prevalence Of Compylobacter Species Amongst Hiv/Aids Patients In Nigeria
}

\author{
Stella Smith ${ }^{1}$, Ngozi Otuonye* ${ }^{*}$, Emmanuel Omonigbehin ${ }^{1}$, Friday Ariyo ${ }^{2}$ \\ and Nkoth Nkoth ${ }^{3}$
}

\author{
Address: ${ }^{1}$ Molecular Biology \& Biotechnology Division Nigeria Institute of Medical Research PMB 2013 Yaba Lagos Nigeria, ${ }^{2}$ Central Public \\ Laboratory Yaba Lagos Nigeria and ${ }^{3}$ HIV Clinic Lagoa University Teaching Hospital Lagos Nigeria \\ Email: Ngozi Otuonye* - mnotuonye@yahoo.com \\ * Corresponding author ‡Presenting author
}

from 2005 International Meeting of The Institute of Human Virology

Baltimore, USA, 29 August - 2 September 2005

Published: 8 December 2005

Retrovirology 2005, 2(Suppl I):P76 doi:10.II86/1742-4690-2-SI-P76

\section{Background}

This study aims to establish the prevalence of campylobacter species associated with diarrhoea among HIV/AIDS patients in Lagos, their prevalent biotypes, antibiotic susceptibility patterns and plasmid profiles. This work was carried out in the Nigerian Institute of Medical Research Yaba Lagos Nigeria between March 2002 to July 2003.

\section{Method}

One hundred and sixty stool and rectal swab samples were collected from confirmed HIV patients seen at HIV clinic of Lagos University Teaching hospital and Central public health laboratory Yaba, Lagos. All the patients were heterosexual with age range between 15 and 55 years. The stool samples were collected from both diarrhoea and non diarrhoea patients. Stool samples were cultured on Butzler's virion medium at $430 \mathrm{oc}$ with oxygen tension of about 5$10 \%$ for 48 hours. The isolates were subjected to ampicillin, cotrimoxazole, tetracycline, erythromycin, ciprofloxacin and gentamycin. Plasmid profile analysis was carried out on Campylobacter jejuni.

\section{Results}

Out of 160 stool and rectal swab samples collected from confirmed cases of HIV/AIDS, 40 isolates were obtained. Campylobacter species were isolated from six $(7 \%)$ out of 84 patients with diarrhea and two $(2.5 \%)$ out of 76 patients without diarrhea. Other bacterial agents included Enterobacter species12 (7.5\%), Salmonella paratyphi A $8(5 \%)$, E.coli $4(2.5 \%)$ followed by Shigella sonnei, Klebsiella pneumoniae, Proteus vulgaris and Candida species. 2 each 1 (25\%). Diarrhoea was more prevalent in males
$120(75 \%)$ than female 40 (25\%). Antimicrobial resistance patterns showed that all the isolates were resistant to ampicillin and cotrimoxazole, only $65 \%$ were resistant to tetracycline and $45 \%$ to erythromycin. All isolates were sensitive to ciprofloxacin and gentamicin. Plasmid profile analysis showed that Campylobacter jejuni isolates lacked detectable plasmids.

\section{Conclusion}

Prevalence of Campylobacter species In HIV/AIDS patients in Lagos was found to be $70 \%$ in patients with diarrhea and $2.5 \%$ in patients without diarrhoea. Campylobacter.jejuni biotype 1 was the most common biotype and gentamicin and ciprofloxacin proved to be the drug of choice for campylobacter species in HIV - infected patients. 